\title{
Técnicas terapéuticas y subjetivación en tratamientos con usuarios/as de drogas
}

\author{
Therapeutic techniques and subjectivation \\ in treatment with drug users
}

\author{
Silvana Laura Garbi ${ }^{1}$ \\ María Cecilia Touris ${ }^{1}$ \\ María Epele ${ }^{2}$
}

${ }^{1}$ Instituto de

Investigaciones Gino Germani, Facultad de

Ciencias Sociales, Universidad deBuenos Aires. U riburu 950/6 ${ }^{\text {to }}$ piso. C1114AAD Ciudad de BuenosAires Argentina. garbisilvana@hotmail.com ${ }^{2}$ Consejo $\mathrm{N}$ acional de Investigaciones Científicas y Técnicas, Universidad de BuenosAires.
Abstract The internment process in therapeutic communities (TC) involves a multiplicity of therapeutic practices and strategies geared to abstinence from drug usage. According to the specialists' own regulations and explicit objectives, the residents must not only abandon the consumption of substances but also adopt new practices, attitudes, emotions and significances through the use of therapeutic techniques that allow them to adapt to the structure of the organization that these institutions impose. Based on the results of the ethnographic survey carried out between 2009 and 2010 in three TCs of the metropolitan area of Buenos Aires, Argentina, the scope of this article is to analyze from a sociological and anthropological standpoint the "therapeutic tools" that comprise the treatment, the subject models that underlie these tools, the consequences that they may produce and their participation in the subjectivity production processes. For this purpose, we focus on analysis of "confrontation" as a privileged and omnipresent strategy of subjectivation in these therapeutic contexts, in order to reveal the epistemological, economic, political and ethical dimensions in the de-subjectivation process of the institutionalized drug user.

Key words Subjectivation, Drugs, Therapeutic tools
Resumen El proceso de internación en comunidades terapéutica (CT) incluye una multiplicidad de prácticas y estrategias terapéuticas orientadas a la abstinencia del uso dedrogas. Deacuerdo a la propia normativa y a los objetivos explícitos de los profesionales, los residentes, no sólo deben abandonar el consumo de sustanciassino también adoptar, a través de la utilización de las técnicas y tácticas terapéuticas, nuevas prácticas, actitudes, emociones, significaciones, que les permita adecuarse a la estructura de organización que imponen estas instituciones. Partiendo de los resultados del trabajo de campo etnográfico realizado entre 2009 y 2010 en tres CT de la región metropolitana de Buenos Aires, Argentina, el objetivo de este artículo es analizar desde la perspectiva de la sociología y antropología crítica las "he rramientas terapéuticas" que componen el tratamiento, los model os de sujetos que subyacen a estas herramientas, las consecuencias que producen y su participación en los procesos de producción de subjetividad. Con este fin, focalizamos en el análisis del "confronto" como táctica privilegiada y omnipresente de subjetivación en estos contextos terapéuticos, a fin de develar las dimensiones epistemológicas, económicas, políticas y éticas en el proceso de des-subjetivación del usuario/ a de drogas institucionalizado.

Palabras clave Subjetivación, D rogas, Herramientas terapéuticas 


\section{Introducción}

El conjunto deestrategiasterapéuticasimplementadas en el dominio de las adicciones se compone por un universo complejo y diverso en el que confluyen diferentes perspectivas teóricas eideológicas orientadas a modificar el consumo de drogas. La mayoría de estas perspectivas comparte el supuesto, más o menos explícito, que modificar el consumo de drogas significa modificar al sujeto. En este sentido, diversas instituciones orientan sus tratamientos a que los usuarios/as de drogas institucionalizados (residentes) no sólo dejen de consumir sino que produzcan transformaciones subjetivas a través de la adopción de nuevas prácticas, actitudes, emociones, significaciones que les permita adecuarse a la estructura de organización que imponen estas instituciones. Esta modificación del sujeto es condición necesaria para dejar el consumo.

Dentro dela variedad demodalidades deatención, la comunidad terapéutica (CT) se constituyeen un privilegiado dispositivo desubjetivación.

A través deun recorrido descriptivo y analítico sobre la multiplicidad de prácticas y estrategias terapéuticas que componen el tratamiento, en este trabajo se evidenciará cómo las técnicas implementadas están íntimamente relacionadas con la concepción que estas instituciones tienen acerca de los usuarios de drogas. Las técnicas y estrategias utilizadas en las CT estarían orientadas a producir transformaciones en los sujetos que les permita, según esta perspectiva, recupe rar la responsabilidad, adoptar otra postura frente a la vida, crear nuevos sujetos.

Enfatizaremos el análisis de las relaciones entre el saber y el poder, entre la epistemología y la política deestastécnicas en el proceso dedes-subjetivación de los residentes. Para ello, nos focalizaremos en la técnica del confronto, es decir, en aquella técnica orientada a que los residentes enfrenten su realidad, sentimientos, pensamientos o comportamientos. Esta técnica, que atraviesa cada una de las herramientas terapéuticas del tratamiento, incluyeun amplio espectro de comunicaciones, diálogos eintervenciones quevan desde la compasión y el más simple interés hasta gritos, denuncias, acusaciones, desafíos, humillación, denigración e insultos. Veremos cómo haciendo uso del confronto se espera que los residentes modifiquen las relaciones previas que tenían con la realidad, los "viejos esquemas" y adquieran una "nueva realidad" que es aportada por otros (residentes, terapeutas). Esta realidad que el confrontado debe reconocer y modificar suele estar aso- ciada a actividades "ilegales", "deshonestas" que adquieren para las CT rasgos y características moralmente considerados negativos.

Cuando hacemos referencia a las técnicas, nos ubicamos en una tradición de pensamiento que arroja luz en los modos en que ciertas prácticas y normativas sociales se corporizan, constituyen y modelan personas y subjetividades ${ }^{1-3}$. En su análisis sobre la inquietud de sí en la Grecia antigua, Foucault ${ }^{3}$ examina y analiza las prácticas sobreel cuerpo y sobre sí en términos de técnicas de subjetivación. En estesentido, la atención, el cuidado y las prácticas - por ejemplo- en la dieta, el sueño, las acciones, el pensamiento y las emociones, son susceptibles deser entendidas como técnicas productoras de sujetos en contextos histórico-políticos determinados.

En su análisis, Foucault ${ }^{3}$ hace del tradicional conócete a tí mismo, una fórmula subsidiaria a la inquietud de sí, que incluye un conjunto heterogéneo de prácticas y normativas sobre la relación del sujeto consigo mismo y con otros. Específicamenteinvolucra normativas y prácticas acerca de los modos en que el poder se relaciona con el saber, entre el gobierno de sí y el gobierno de y por partedeotros, en tre conocimiento y dominación.

Siguiendo esta tradición analítica, el análisis del confronto, nos permitirá develar las dimensiones epistemológicas, económicas, políticas y éticas en el proceso de des-subjetivación de los residentes al mismo tiempo que entender queen cuanto técnica, no sólo modela cuerpos, define moralidades y diferencia sujetos sino quese constituye como táctica privilegiada y omnipresente de subjetivación en estos contextos terapéuticos.

\section{Métodos}

El trabajo de campo del presente estudio se realizó en tres CT (dos de varones, y una de mujeres) de la región metropolitana de la provincia de Buenos Aires, Argentina.

Con el propósito de analizar críticamente las herramientas terapéuticas que componen el tratamiento y la función quedesempeñan en el proceso de producción de subjetividad, seha utilizado el método etnográfico prevaleciendo las técnicas de observación participante (registradas en notas de campo), entrevistas en profundidad (grabadas y desgrabadas) y conversaciones informales con residentes y profesionales. Se realizaron un total de 25 entrevistas a residentes varones y mujeres. En las sedes de varones se realizaron 18 entrevistas a residentes de entre 18 y 50 
años con una media de 24 años de edad. En la sede de mujeres se llevaron a cabo 7 entrevistas en profundidad a residentes de entre 18 y 30 años. En esta sede, la muestra total arrojó una media de 22 años de edad. También se realizaron 10 entrevistas a profesionales de distintas disciplinas (psicólogos, psiquiatras, sociólogos, operadores terapéuticos, directores de comunidades terapéuticas) de diversas instituciones nacionales, municipales y asociaciones civiles relacionadas directa o indirectamente al área de adicciones. La investigación se llevó a cabo de acuerdo con las normas establecidas en la Declaración de $\mathrm{H}$ elsinki. En todas las entrevistas realizadas se contó con el consentimiento informado de los entrevistados a través del cual se explicitó los propósitos del estudio y la metodología aplicada y se informó acerca del carácter voluntario, confidencial y anónimo de la entrevista. En consecuencia, como se estila en la investigación cualitativa, los nombres de todos los entrevistados se han modificado por otros de fantasía para proteger su identidad y se han borrado de las entrevistas todo elemento y referencia que permita identificar a los participantes de modo directo e indirecto.

Tomando en consideración queúnicamentea través de mirar, escuchar, preguntar, formular hipótesis y cometer errores el etnógrafo puede adquirir un conocimiento sobre la estructura social del lugar y comenzar a entender la cultura de los miembros del grupo ${ }^{4}$ nos fue imprescindible para esta investigación, participar de la estructura diaria en la que se desarrolló el tratamiento.

Fueasí que, la complementariedad de las técnicas metodológicas utilizadas nos ha permitido interpretar de forma más acabada cada una de las dimensiones que conforman el tratamiento, permitiéndonos identificar la postura epistemológica desdela cual trabajan los equipos terapéuticos y quevuelveinteligible el tipo detratamiento planteado.

En la escritura se han utilizado diferentes recursos estilísticos para señalar diferentes tipos de textos y/o expresiones: cursiva (nociones teóricas), comillas (para diferenciar términos 0 expresiones pertenecientes a otros discursos: términos nativos, teóricos de otras disciplinas, fragmentos de entrevistas y notas de campo.

\section{Resultados y discusión}

Fundamentos de la Comunidad

Terapéutica (CT)

Las CT pueden ser pensadas en términos de lo que Erving Goffman ${ }^{5}$ definió como institución total, es decir: a diferencia del ordenamiento social básico, dondeel individuo tiende a dormir, jugar y trabajar en distintos lugares, con diferentes coparticipantes, bajo autoridades diferentesy sin un plan racional amplio, las instituciones totales se presentan como una ruptura ante estas situaciones: en todos los aspectos de la vida se desarrollan en el mismo lugar y bajo la misma autoridad única; cada etapa de la actividad diaria del miembro se lleva a cabo en la compañía inmediata de un gran número de otros; todas las etapas de las actividades diarias están estrictamente programadas; finalmente las diversas actividades obligatorias se integran en un solo plan racional, deliberadamente concebido para el logro de los objetivos propios de la institución.

Toda CT se organiza mediante una "estructura" altamente reglada y normatizada que regula el empleo del tiempo y espacio. Nada queda sujeto al azar: con el objetivo de que el residente logre introyectar y respetar un orden en su vida cotidiana, todo se organiza bajo un mismo cronograma de actividades. Para lograrlo se recurre a un sistema de control permanentea través de la distribución piramidal delasfunciones y la verticalidad del poder: en el vérticesuperior seubicael "staff" (compuesto por el director, vicedirector, profesionales, administrativos, operadores socioterapéuticos y terapeutas) mientras queen la base se ubican los "residentes", quienes pueden asumir diversos roles y/o posiciones de acuerdo a su adaptación al tratamiento. La estructura vertical facilita el sistema de obediencia demando, y medianteun mecanismo de premios y castigos, tanto morales, psicológicos como materiales y al gunas veces físicos, se intenta promover un "aprendizaje" en el residente ${ }^{6 .}$ Para lograr estos objetivos, las CT generan un dispositivo de internación prolongado (de 1 a 3 años) que puede ser a "puertas abiertas" o a "puertas cerradas".

Una delas características principales de las CT es la utilización de la comunidad como método: se parte de que a través de la interacción estructurada y no estructurada, los residentes pueden influir mutuamenteen las actitudes, percepciones y comportamientos asociados al uso de drogas.

Es por ello que el tratamiento se basa en actividades grupales y cada residente tiene una parti- 
cipación activa en su propio tratamiento y en el de sus compañeros. En las CT no solo se prioriza el cambio de conducta, sino que también una conversión moral del sujeto, es decir "el crecimiento de los residentes como personas", "sus valores humanos y su capacidad de amarse y amar responsablemente". Es así que, sostener un "amor responsable", abogar por la "no violencia", "no alcohol y no drogas" constituyen las reglas primordiales de las comunidades terapéuticas?.

En las CT, la transformación subjetiva se constituye en el lei v motiv del tratamiento. La ideologíaquesustentan estasinstituciones tieneque ver con entender quela abstinencia en el consumo de drogas supone un transformación subjetiva, es decir, la adopción de nuevos valores y el cambio de hábitos de vida (que de acuerdo a esta perspectiva conducea los sujetos al consumo de drogas y los exponen a otros riesgos asociados al consumo). Tras la entrevista de admisión y el diagnóstico que realiza el profesional sobre la personalidad y la patología del residente, la me cánica del tratamiento resideen la incorporación de las normas, valores y actitudes propuestas por la institución. Deesta manera, el equipo tratante evalúa periódicamentela "evolución" de los residentes que se concreta en los pases de fases, hasta obtener el alta, y con ella la libertad. Este tipo de tratamiento "progresivo" recibe críticas en al menos dos aspectos centrales. El primero sostiene que a través de un "programa de cura tipo" se insta a dejar de lado otro tipo de terapéuticas puesto que suponen un ideal de intervención universalizante centrado en el rasgo común que identifica a los residentes (la adicción a las drogas), en el cual un único método resulta eficiente para todos ${ }^{8}$. Este método se vuelve posible tras asumir quetodo aquel que consumió drogas atravesó por situaciones análogas, o adoptó actitudes similares. Es decir, por relacionar el uso de drogas con un tipo de personalidad.

Otra crítica tiene que ver con considerar que la adaptación y el buen comportamiento en el interior de las instituciones de encierro es extrapolable a las condiciones de su medio cotidiano (su barrio, su entorno vincular, etc.) ${ }^{9}$.

\section{Herramientas terapéuticas}

Si bien las instituciones pueden variar entre sí, el uso de las herramientas terapéuticas es similar, dado que están basadas en la misma imagen de sujeto como "adicto".

Entre las herramientas terapéuticas tienen mayor preponderancia aquellas deíndolegrupal, entre ellas podemos mencionar: los "grupos de apertura" y "cierre" que se realizan con todo el grupo de residentesy consisten en plantearsecuáles serán los objetivos personales y grupales que se pretenden cumplir durante el transcurso del día; el "dinámico" que seutiliza para queentrelos residentes se señalen las actitudes que consideran impropias dentro del tratamiento. Los operadores terapéuticos consideran que esta técnica funciona como un "espejo" para mostrar quédel otro es al go delo propio. El objetivo es queel residente asuma la responsabilidad (en tono de culpabilidad) de todo lo que ledicen, esté o no de acuerdo y secomprometa a "revertir" la situación; la "asamblea" funciona como un espacio en el que seespera que se "suspendan" las jerarquías durante el tiempo que dura el grupo, es decir, los residentes pueden cuestionar, reclamar, requerir explicaciones, sobre cuestiones relativas al tratamiento.

Esta sumatoria de técnicas y estrategias utilizadas en la CT están orientadas a producir transformaciones en los sujetos queles permita, según esta perspectiva, recuperar la responsabilidad, tener otra postura frente a la vida. Estos objetivos son reforzados a través de la "filosofía" que afinca la identidad institucional. La falta decompromiso con el cambio, la desobediencia, la inadaptación a la estructura de la CT son encauzadas a través de las herramientas terapéuticas, sobre todo a aquella que subyace todas las herramientas y queresulta la técnica por excelencia: el confronto.

\section{El Confronto}

La técnica del confronto surge a partir de que en 1925 comienza a asociarse a los usuarios de drogas con una "personalidad defectuosa", con una historia prolongada de "inadaptación social" y atrapado en subculturas "desviadas" 10 . Este viraje facilitó la emergencia de nuevos dispositivos de tratamientos orientados a mejorar la "maduración personal y los valores pro-sociales" de los pacientes. Sesuponía que un ajustesocial librede drogas en la institución lograría la adaptación del adicto a los cánones considerados socialmente aceptables. El fracaso de esta hipótesis, confirmada por los informes de el evadas tasas de recidivas después del alta, creó un clima de frustración que fomentó la búsqueda de tratamientos alternativos que incluyeron, entre otras, terapias de confrontación ${ }^{11}$. Entre la década del 40' y 50' Tiebout, desde la psiquiatría, sentó las bases de la terapia de la confrontación en el tratamiento de "alcohólicos" a partir de considerar que de- 
bían su adicción a una "malformación de carácter". El "alcohólico", en su opinión, era incapaz de una autopercepción exacta de si mismo y de los demás, debido a un complejo sistema de mecanismos de defensa (por ejemplo, negación, proyección dela culpa) medianteel cual reforzaba su autoestima al tiempo que justificaba su necesidad de beber. Desdeesta perspectiva el tratamiento debía ir en la línea de lograr un proceso de rendición deestas actitudes, la reconstrucción de la personalidad y el desarrollo de una forma disciplinada de vida. Estas concepciones fueron ampliamente aceptadas eincorporadas primero por los grupos de Alcohólicos Anónimos (AA) y luego por las primeras $\mathrm{CT}^{11}$. Fue así como a partir de los años 60 las terapias de confrontación se difundieron en el tratamiento para las adicciones a las drogas ${ }^{12}$.

En relación con las $\mathrm{CT}$, algunos autores han caracterizado a la técnica de confrontación como "una forma distinta y específica de ayuda hallada en la comunidad terapéutica", "un tipo especial defeed back social", "el espejo quemedevuelvela imagen de quién soy, de cómo estoy, de cuál es la consecuencia de lo que hago"13. Esta técnica es considerada como una "herramienta axial" de la comunidad, a través de la cual los residentes son implacablemente confrontados por sus pares a cada instante "enfrentando la mentira, la proyección de la culpa y la justificación"7. Esta técnica es presentada como la vía más directa para la "ruptura de las defensas" ya que "nadie puede engañarse a sí mismo y a todo un entorno vigilantey alerta que está viviendo o queha vivido lo mismo"13. Por ello se considera que es a través de la confrontación que se logra eliminar la"(auto) imagen de la calle", que se logra la conexión con los propios sentimientos y, que es la vía más estratégica para que los residentes acepten las normas dela CT: "o acepta lo quese le dice y cambia, o se va de la comunidad"13. En este orden de ideas, la confrontación sirve para "tomar conciencia de la enfermedad", y para saber que las acciones y/u omisiones lo afectan a uno mismo y a los demás ${ }^{6}$.

Llegados a este punto, debemos considerar quesi bien esta técnica incluye un amplio espectro de comunicaciones, diálogos e intervenciones, quevan desdela compasión y el más simple interés hasta gritos, denuncias, acusaciones, desafíos, humillación, denigración einsultos cuando se habla del uso de la confrontación en los tratamientos de adicciones en general y de drogas en particular, se hace referencia específicamentea aquella clase deintervenciones y devolu- ciones vinculadas con las sanciones, denuncias y humillaciones. En estesentido la aparición detécnicas de confronto podría haber sido considerada como una contratransferencia fuera de control, y de violación de la ética profesional sin embargo es considerada no sólo como un enfoque necesario para el tratamiento sino "como el único idioma que pueden entender"11.

Bases epistemológicas del Confronto como técnica de des-subjetivación

Interrogar el conjunto de técnicas terapéuticas de confrontación, es ingresar a un universo complejo y diverso donde convergen perspectivas teóricas e ideológicas, prácticas terapéuticas, moralidades, normativas y modalidades de subjetivación orientadas a modificar el consumo de drogas. Aún reconociendo esta diversidad, la mayoría de estas perspectivas considera que el problema de la adicción no se puede abordar de forma puntual ni aislada. Es decir, estas prácticas y/o comportamientos que se clasifican como "adicción", en este caso a las drogas, no pueden ser disociados de la "personalidad", "Ios hábitos", "Ia "conducta", o la subjetividad previa. Por esta razón, modificar el consumo supone modificar al sujeto. "Quitar las imágenes" que traen los residentes, aquellas características que van constituyendo su identidad, se convierte en la parte neurálgica de la des-subjetivación: “la imagen es la ropa, la forma de caminar, el vocabulario, las miradas, todo lo que los lleva a situaciones de consumo en la calle. Cuestiones quetratamos lo mejor posible de quitarles, ¿no?"(Vanesa, O peradora Terapéutica)

Las técnicas y tácticas terapéuticas, entre ellas el "confronto" son herramientas en esta transformación, con las que de modo variable y de acuerdo a las orientaciones, se debe des-subjetivar. Bajo esteobjetivo, el tratamiento en sí seconstituyeen una estrategia a partir dela cual poder y saber se implican mutuamenteen la producción de subjetividad.

Los modos dominantes de tratamiento para las adicciones suponen, en la mayoría de los casos reducir la diversidad de los sujetos a la homogeneidad del sujeto "adicto", cuyas características definitorias se entrelazan y fundamentan mutuamente con aquellas características que tienen los tratamientos. Este ensamblaje no solo otorga inteligibilidad y legitimidad mutuaa la constitución simultánea del sujeto "adicto" y de su tratamiento, sino también a la producción de normativas institucionales y prácticas terapéuticas. 
Uno de los mecanismos para homogeneizar las diferencias es por ejemplo anular en relación al consumo los atravesamientos barriales, culturales, económicos, sociales, entre otros. Así lo refleja el siguientefragmento: "(hay residentes que consideran que) los demás no son nada (y se preguntan) ien qué me voy a identificar yo con éste pibe?Y en realidad tienen un montón decosas en común (es decir) vos estás acá porque sos adicto igual que él, no lo pudiste manejar, te trajo un montón de problemas a nivel de relacionarte socialmente, o sea, por ahí con diferenteconcepto 0 de diferente manera, pero en realidad la base del conflicto es la misma" (Vanesa, operadora terapéutica)

Otro delos mecanismos de homogeneización es considerar quetodo consumo es abusivo, problemático, anulando otras modalidades como puede ser el uso recreativo, social: "acá te dicen que la ilusión de control no existe, que es una pura excusa que hace tu cabeza, o dicen queellos no conocen a nadie que consuma con éxito (en forma controlada); (Pero) Yo sí y dentro de mi familia hay una hermana que consume con éxito hace muchos años, estudió la carrera de psicología, se recibió con un excelente promedio, trabaja en lo que le gusta y consumió drogas toda su vida. Qué sé yo, capaz que ella puede manejar el consumo y yo no, no sé, pero hay consumidores sociales. Acá (es) como que lo que te dicen ellos es la verdad absoluta" (M arcos, Residente).

Desde esta perspectiva, y bajo estas circunstancias se pronostica un final que siempre está vinculado a lo trágico: "(la adicción) es incurable, progresiva y mortal. (...) todos empiezan probando y terminan con diez gramos de cocaína, hechos mierdas o en el cementerio, en la cárcel 0 postrado en una silla de ruedas. Otro final no tiene la adicción. Y hay una cuarta opción quees la rehabilitación" (José, O perador terapéutico)

La técnica del confronto involucra diferentes dimensiones dela realidad y vida cotidiana: epistemológicas, económicas, políticas y éticas. En primer lugar, en el confronto se asume cuestiones acerca de la real idad, la negación, la verdad, el saber, el conocer, y reconocer por parte de los usuarios/as de drogas, es decir, se juega una dimensión epistemológica.

En epistemología o también llamada filosofía de la Ciencia, la confrontación involucra complejas relaciones entre el saber y la realidad que hacen queel conocimiento científico tenga un estatuto diferente de la metafísica y del sentido común. Los tratamientos que involucran el confronto o por involucrar al confronto en los tra- tamientos, se producen ciertas asunciones sobre las relaciones entre el sujeto, la realidad y la verdad. Más precisamente, asumen que la adicción suponeuna torsión de estos vínculos, o la distorsión de estos vínculos promueven la adicción.

Para que el confronto sea terapéutico debe modificar las relaciones previas que el sujeto tiene con la realidad.

“Evasión”, "distorsión”, “negación”, “deshonestidad", "mentira", son al gunos de los cal ificativos para dar cuenta de las relaciones entre el "adicto" y la "realidad", vínculos en que la "verdad" se ve comprometida. Poco importa las direcciones y orientaciones de causas y efectos. La técnica debe actuar sobre esta dimensión, transformarla, para que el sujeto asuma la "verdad", se confronte con la "realidad", modifique sus actitudes a través del claro conocimiento de sí, aportado por otros: "me gritaban que era mentira que quería a mi hijo porque decían que si yo realmente quería a mi hijo no me iba a estar acostando con cualquiera. A mí me dolía pero después entendí que era cierto" (Carla, residente).

El confronto, involucra a otros que intervienen en el conocimiento y el reconocimiento dela verdad por parte del "adicto". El conocimiento sobre el que se habla, en general no es neutro ni inocuo, involucra aquellos rasgos o características morales "negativas", "ilegales", "deshonestas" y "estigmatizadas" como por ejemplo tener "actitud cómoda"; "antihigiénica"; "no tener amor responsable"; "usar el léxico dela calle"; "cuestionar los límites"; "ser soberbio". Estas actitudes a confrontar son las que de acuerdo a la institución caracterizan a los sujetos como "adictos". Ello se desprende de la siguiente nota de campo: Con el objetivo de confrontar a un residente para "ayudarlo porque vemos que no está haciendo nada por él" los operadores terapéuticos hacen uso de una herramienta terapéutica a través del cual toda la casa comienza a confrontar sus actitudes negativas que se despliegan una tras otras sin respiro y sin la espera de una respuesta, por el tiempo que demande (que puede llegar a una hora). Así lo expresa un residente: “D epende de lo que sepamos deél le damos con un caño: ipor qué no aprovechás el tratamiento?; ¿por qué sos tan irónico?; ¿por qué sobrás a la gente?; ¿Por qué te das permiso? y también cosas de su vida ipor qué no te hacés cargo de tu papá?; ipor qué hacías prostituir a tu mujer? Y así pim, pam, pum pero sin dejar que responda para que se confunda y reaccione ¿entendés?" (M anuel, Residente)

Este tipo de confrontaciones lleva a confundir, a desestructurar al sujeto. Al no dar lugar a la 
réplica, el confrontado debe asumir sin más todo lo "malo" que se dice de él, pero además debenecesariamente modificar la actitud confrontada 0 al menos fingir haberla modificado- si no, tanto los pares como los terapeutas volverán a confrontarlo las veces que sea necesario hasta que logre esa transformación. Así es explicado cómo se confronta una actitud: "cambialo, cambialo y así hasta que no lo cambiás no te vas a dejar de sentar en esa silla, quees un garrón sentarteen esa silla y que te caguen a gritos" (Carlos, Residente)

Restringida en un principio a relaciones entre pares, el uso del confronto fue integrado también en relaciones asimétricas, específicamente operadores y terapéutas. Para que pueda promover la transformación subjetiva, el confronto atraviesa todo el espectro de vínculos y áreas de la vida cotidiana. Por esta razón, las técnicas confrontacionales obligan y hasta en algunos casos buscan doblegar, quebrar a las personas, como condición necesaria para que conozcan y reconozcan estas realidades y verdades.

Esta búsqueda de quebrar a la otra persona está vinculada con la premisa teórica de estas terapias catárticas que entienden que un amplio espectro del sufrimiento humano tienesus raíces en los sentimientos reprimidos que pueden ser aliviados mediante técnicas que faciliten la liberación emocional. La confrontación verbal resultó, para estas terapias el medio más eficaz de la participación y el cambio de conducta adictiva ${ }^{11}$ : "capáz que al otro vos le hablás, le hablás y sigue igual (entonces) lo agarrábamos entretres, le gritábamos, descargábamos todo lo que pensábamos y lo que sentíamos y me metía en la familia y me metía en los sentimientos de él, del padre, del hermano, lo que fuere, hasta que lo hacía quebrar, ¿entendés?

- ¿Ese era el límite?

- Claro, esos eran como los métodos que utilizábamos para poder ayudar y para ayudarse uno mismo, porque a mí también me hacía bien" (Gustavo, Residente)

En los relatos es muy habitual encontrarse con esta noción de ayudar y ayudarse a través del espejo con las actitudes del otro. El problema radica cuando esta "ayuda" está íntimamente re lacionada con el objetivo de quebrar a la otra persona: "por más que acá te digan que lo que te perjudica te ayuda yo creo que no, que lo que te perjudica, te perjudica, y meestás perjudican do y me estoy resintiendo, ¿entendés? porque me estás cagando a pedos, me estás haciendo hacer cosas que no quiero y me estoy enojando con vos, no es que me estás ayudando como me de- cís, ¿viste?' (M arcos, residente); 'a mí loco meverdugueaban en la calle, no vengo acá para ser verdugueado. Yo vengo acá paraque meayuden ¿entendés? A mí ya me verduguearon bastante, la policía, en el penal, en muchos lados, no quiero que me verdugueen en la comunidad terapéutica" (Carlos, residente)

En este doblegar, quebrar al otro, desestabilizarlo es donde la dimensión de poder que sejuega en el confronto se hace visible y evidente. Es decir, donde la técnica se convierteen una táctica de poder.

Por esta singular combinación, el confronto tiene propiedades paradojales que generan una suerte de profecía auto-cumplida. Partiendo dela perspectiva quesostienequeel "adicto", como constructo homogéneo, tiene determinadas relaciones con la realidad y la verdad, este conjunto de técnicas y tácticas confrontacionales termina produciendo, en su mismo desarrollo, el tipo de sujetos quebusca erradicar. Asumien do por principio que los adictos "mienten", son "deshonestos" o "vagos", con frecuencia se busca o se fuerza que los adictos reconozcan que están "mintiendo", son "vagos" o no asimilan la "realidad" como parte inherente de las prácticas cotidianas y terapéuticas. La búsqueda de la terapéutica opera en el pasaje que deben lograr los residentes del "control" al "autocontrol": "lo quetiene el dinámico es quevos legritás a la persona lo quete parece, son varias actitudes que vos le decís, no? A cá te dicen que si una es verdad, vos te tenés que hacer cargo de todas, ¿entendés? y capaz que nada que ver, y como tedijeron una queera verdad, vos tenés que decir todas que sí, y es horrible.

- ¿Y qué pasa si decís que no te hacés cargo de determinadas actitudes que te remarcaron?

- Y si decís que no, como que se pudre todo, te tenés que hacer cargo sino por ahí sacan a todos a baldear la casa" (M arcos, Residente).

De esta manera, se fuerza a mentir y a ser deshonesto (es decir, asumir las mismas actitudes que el tratamiento pretende modificar) haciéndose cargo de actitudes que no consideran tener sin la posibilidad de defenderse 0 excusarse.

Lo mismo sucede cuando el residente no se acerca al estereotipo creado por la CT: aquel que tiene un pensamiento crítico es compelido a través de estos mecanismos a adoptar las actitudes propias del estereotipo: "me dicen que por haber estudiado psicología, utilizo eso para creerme mejor que las chicas. Así me colocan en un lugar, meetiquetan y siguen sin escucharme, como afue ra. Eso no meayuda, entoncestengo quedejar de hablar, de pensar (entonces) ino puedo utilizar 
lo que sé?' (Eugenia, Residente); “no podés pensar diferente a lo que piensa el grupo porque te van a avasallar de todos lados. el staff me dice quea mí la inteligencia se me pone en contra, en el único lugar donde me pueden decir esas cosas es acá, ¿entendés? dicen que al ser inteligente le buscás mucho la vuelta, son cosas queyo no comparto mucho, pero sabés que no podés expre sarlo, porque te sancionan. Pero bueno, ya está, estoy acá adentro, estoy en el baile, hay que bailar' (M arcos, Residente)

En este sentido "estoy en el baile, hay que bailar", supone adoptar ciertas estrategias adaptativas que le permiten al residente atravesar, con "éxito", el tratamiento. Esto implica que al ser observados, los residentes hagan lo que se espera de ellos, finjan adoptar actitudes, sentimientos, acciones acordes a las promovidas por los tratamientos. Desde la perspectiva del interaccionismo simbólico, Ervin Goffman ${ }^{14}$ señala que toda interacción social constituye una actuación (performance), es decir, un papel representado anteuna audiencia. En estesentido Goffman considera que cuando sepresenta en público, el sí mismo de las personas (self), es el resultado de un proceso denominado "manejo de impresiones" (impressions managment) a través del cual controla las impresiones que seforman deél y las clases de cosas queél puedey no puedehacer mientras realizasu representación, con la finalidad de elaborar una imagen de sí mismo conforme a los deseos del otro (de la audiencia) ${ }^{14}$. En una CT, representar una buena imagen de sí mismo es condición para obtener el alta. Al ser la conducta observable un indicador de la transformación subjetiva, es esperableentonces, que éstos presenten asu sí mismo, y si es necesario, simulen su comportamiento $o$ falseen su conducta frente a cual quier observador con capacidad o influencia en su evaluación operando funcionalmente lo que Gustavo Hurtado denomina obediencia fingida ${ }^{8}$.

$\mathrm{H}$ aciendo un paralelo con el tratamiento en el contexto penitenciario, Adelantado ${ }^{15}$ a propósito de analizar los programas de intervención penitenciaria en Cataluña sostenía lo siguiente: "el objetivo manifiesto de la institución es cumplir el mandato legal resocializador, aunque el objetivo latentees el mantenimiento del orden interior. (...) La conformidad aparece como una forma de ne gociación entre el interno y la institución sobrela forma de definir la realidad; pero frente a la distribución asimétrica del poder y de la definición sobre la necesidad y condiciones de la reeducación, los internossedefienden con la desidia frente a las actividades propuestas que no responden a sus intereses, o bien, manteniendo sus hábitos deconducta y su moral de forma clandestina. Esa resistencia a la influencia es lo que se llama 'conformidad simulada', que consiste en aceptar de forma pública un comportamiento o un sistema de valores sin adherirse a ellos de forma privada (conversión o internalización)"15.

Pero aún sosteniendo que la conducta de los residentes no sea simulada, es necesario reparar en que la evaluación se refierea su conducta dentro del ámbito institucional, es decir, se evalúa su conducta en función de si se adaptó o no a las normas de la "casa" más allá de considerar el ámbito cotidiano donde seguirá desarrollando su vida una vez que obtenga el alta.

Es dable pensar que esta falacia de considerar quetal adaptación es extrapolable a las condiciones de libertad termine promoviendo otra de las profecías autocumplidas: la el evada tasa de reincidencia en el consumo: "hace tiempo que sefueron diez de alta y recayeron los diez, ¿entendés? Es como que hay muchísima falla, chicos que han estado un año acá y al día recayeron y están internados otra vez, y digo, ¿cómo puede ser?"(M arcos, Residente)

Sin un replanteo profundo de las bases sobre las que se sustenta ésta táctica, la escasa eficiencia de estas instituciones seguirá estando limitada a terminar con éxito el tratamiento, un éxito que como dijimos sólo remite a un "saber actuar".

\section{Consideraciones finales}

En esteartículo hemos puesto en evidencia cómo el confronto como técnica de subjetivación, traza cuestiones atinentes no sólo a las terapéuticas, sino también a las moralidades. Este proceso suponeque una vez que el residente, ha adquirido la "conciencia de enfermedad" y está dispuesto a curarla, debe interiorizar su "nueva imagen" y debe asumirse como "adicto", con todos los rasgos que esta identidad supone. Este estereotipo condena al usuario/a a llevar este estigma durante el resto de su vida. Esta situación además, se convierte en paradojal considerando que se ingresa a una institución de salud para curarse de un estado que la propia institución califica como "incurable".

Según Castel y Coppe ${ }^{16}$, gracias a esta teoría los usuarios/as admiten su peso, se reconocen vencidos por el producto y con una personalidad endeble, siempre tentados a perder la voluntad desostener el autocontrol. En estesentido, se desculpabilizan por su debilidad, y asumen vice- 
ralmente que deben vivir con ese estigma y en abstinencia, sin el producto de su adicción que siempre los tienta a volver ${ }^{15}$. La fuerza de esta creencia ha supuesto entonces, la asunción de la "enfermedad", la necesidad de la "cura", el pasaje por el tratamiento de "desobjetivación" y la adquisición de una "nueva identidad" para toda la vida. Deestemodo, "Ios residentes realizan el tratamiento para recuperar "su voluntad" frente a la "tentación", así como la capacidad para tomar decisiones con responsabilidad"17.

El problema del poder en las prácticas terapéuticas no puede excluir el problema de la desigualdad y de las diferencias sociales. En el caso que nos ocupa, la mayoría de las personas institucionalizadas en las comunidades terapéuticas investigadas provienen de poblaciones marginalizadas de la Ciudad y del Gran Buenos Aires lo que hace insoslayable analizar el confronto en articulación, relación y correspondencia con otras técnicas y prácticas que tienen lugar por fuera de los contextos terapéuticos. Aún cuando son reconocidas como un legado de otros países y de diferentes orientaciones terapéuticas, el análisis del confronto no puede desconocer la existencia deun conjunto de prácticas locales de des-subjetivación que atraviesan diversas instituciones y áreas de la vida cotidiana.

La mayoría de las personas consumidoras de drogas que provienen de estas poblaciones han tenido otras experiencias con prácticas y técnicas afines, semejantes o parcialmente relacionadas por origen en institutos de menores, comisarías, cárceles, incluso en algunos casos en hospitales y/o en sus propias familias ${ }^{18}$. Estas instituciones, a través de sus prácticas discursivas y extradiscursivas, se constituyen en dispositivos a través de los cuales se instrumentaliza el gobierno de las poblaciones vulnerables ${ }^{19}$. M ediante -en apariencia- distintas tecnologías de poder se busca normalizar lo desviado, en su paso por los distintos dispositivos de control. En el caso de las adicciones, la normalización, para losmodos dominantes de los tratamientos supone, en la mayoría de los casos reducir la diversidad de los sujetos a la homogeneidad del sujeto "adicto". Uno de los mecanismos para homogeneizar las diferencias es por ejemplo anular en relación al consumo los atravesamientos barriales, culturales, económicos, sociales, entre otros. De esta manera, se incluyen dentro demodelos homogéneos, consumo, prácticas, trayectorias, sustancias y ritmos por completo heterogéneos. Una vez configurado al "adicto" como constructo homogéneo, se despliega una serie de técnicas de control que tienden a la normalización y producción de nuevos sujetos. Es posi ble reconocer en estas técnicas, presentes en los más variados dispositivos por los que atraviesan las personas que consumen drogas y provienen de las poblaciones más vulnerables, los modos de gobernar a dichas poblaciones.

En este sentido, el análisis del confronto no puede dejar de señalar la necesidad de realizar un estudio posterior de las características de la dispersión social deesteconjunto diverso de técnicas confrontacionales (en interrogatorios, castigos, etc.). Desdeesta perspectiva, el confronto sevecuestionado también en su aparenteoriginalidad y por lo tanto en su eficacia terapéutica para producir una transformación subjetiva que recale en una mejoría para el usuario/a. Estas técnicas no sólo estarían en sintonía con experiencias y vínculos ya existentes en estas otras instituciones sino también las perpetúan y profundizan en el tiempo.

\section{Colaboradores}

SL Garbi, M C Tourisy M Epelehan desarrollado las siguientes actividades para ser consideradas como tales: contribuyeron al desarrollo de ideas y diseño para el estudio: realizaron la recolección de datos, al igual quesu análisis einterpretación; redactaron el artículo, revisaron y aprobaron la versión final a publicarse. 


\section{Referencias}

1. M auss M. Técnicas y movimientos corporales, Sociología y Antropología. Madrid: Ed. Tecnos; 1971.

2. Bourdieu P. El sentido práctico. Madrid: Siglo Veintiuno Editores; 2007.

3. Foucault M. Historia de la sexualidad 2. El uso de los placeres. Madrid: Siglo Veintiuno Editores; 2005.

4. Hammersley M, Atkinson P. Etnografía. M étodos de investigación. Barcelona: Ed. Paidós; 1994.

5. Goffman E. Internados. Ensayos sobre la situación social de los enfermos mentales. Buenos Aires: Amorrortu; 1961.

6. Secretaría de Programación para la Prevención de la Drogadicción y la Lucha contra el Narcotráfico (SEDRONAR). Las comunidades terapéuticas. Revista Hablemos 2005.

7. Danza C. ¿Tratable o intratable? La comunidad terapéutica para drogodependientes, una alternativa. Revista de Psiquiatría del U ruguay 2009; 73(1):99-108.

8. Hurtado G. Las toxicomanías y los dispositivos de obediencia fingida. En: Dobón J, Rivera Beiras I, coordinadores. Secuestros institucionales y derechos humanos: la cárcel y el manicomio como laberintos de obediencias fingidas. Barcelona: Ed. M aría Jesús Bosch SL; 1997. p. 215-220.

9. García Borés Espí P. La evaluación psicológica en las penas privativas de libertad. En: Dobón J, Rivera Beiras I, coordinadores. Secuestros institucionales y derechos humanos: la cárcel y el manicomio como laberintos de obediencias fingidas. Barcelona: Ed. M aría Jesús Bosch SL; 1997. p. 151-165.

10. Acker C. Creating the American junkie: Addiction research in the classic era of narcotic control. Baltimore: Johns Hopkins University Press; 2002.

11. White W, Miller W. The use of confrontation in addiction treatment: History, science and time for change. Counselor 2007; 8(4):12-30.
12. Forrest G. Confrontation in psychotherapy with the alcoholic. Holmes Beach: Learning Publications; 1982.

13. Goti ME. La comunidad terapéutica. Un desafío a la droga. Buenos Aires: Nueva Visión; 1990.

14. Goffman E. La presentación de la persona en la vida cotidiana. Buenos Aires: Amorrortu; 2009.

15. Adelantado G. Orden cultural y dominación. La cárcel en las relaciones disciplinarias [tesis]. Departamento de Sociología de la Universidad Autónoma de Barcelona; 1992.

16. Castel R, Coppel A. Los controles de la toxicomanía. En: Ehremberg A. Individuos bajo influencia: drogas, alcoholes, medicamentos psicotrópicos. Buenos Aires: Nueva Visión; 2004. p. 221-238.

17. Renoldi B. Estar sano es ser persona. El caso de los usuarios en tratamiento. Buenos Aires: Universidad de Buenos Aires; 2001. (Cuadernos de Antropología Social, n. 13)

18. Epele M. Sujetar por la herida: una etnografía sobre drogas, pobreza y salud. Buenos Aires: Ed. Paidós; 2010.

19. Foucault M. Seguridad, territorio, población. Buenos Aires: Fondo de la Cultura Económica; 2006.

Artigo apresentado em 13/04/2011

Aprovado em 14/05/2011

Versão final apresentada em 06/07/2011 